\title{
18F-FDG PET/CT as a modality for the evaluation of persisting raised infective markers in patients with spinal tuberculosis
}

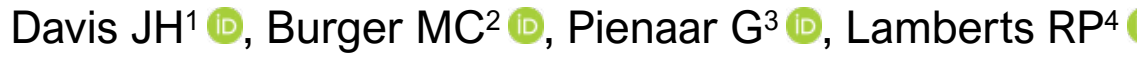 \\ $1 \mathrm{MBChB}, \mathrm{FC}$ Orth(SA), MMed(Orth)(SA); Consultant orthopaedic surgeon, Spinal surgery \\ 2 BSc, B(Med)Sc Hons (Med Virol), M(Med)Sc (Med Virol), PhD (Exercise Science); Lecturer/Scientific research coordinator \\ 3 MBChB, FC Orth(SA), MMed(Orth)(SA); Consultant orthopaedic surgeon \\ 4 MSc (Med HMS), PhD (Exerc Physiol), FECSS; Professor and Head of Research
}

Division of Orthopaedic Surgery, Department of Surgical Sciences, Faculty of Medicine and Health Sciences, Stellenbosch University, Tygerberg, South Africa

Corresponding author: Dr Johannes H Davis, Division of Orthopaedic Surgery, Department of Surgical Sciences, Stellenbosch University, Tygerberg Campus, PO Box 19063, Tygerberg, 7505, South Africa; tel: +27 (0)21 938 9266; email: johanhdavis@gmail.com

\begin{abstract}
Aims: The aim of the study was to investigate the differences in participant characteristics between positive and negative, positron emission tomography with 2-deoxy-2-[fluorine-18]fluoro-D-glucose integrated with computed tomography (18F-FDG PET/CT) activity at the spinal tuberculosis (TB) site following 12 months of the appropriate chemotherapy therapy for spinal TB. A secondary aim of the study was to determine whether erythrocyte sedimentation rate (ESR) levels could be used as a reliable marker of TB activity and/or treatment success of spinal TB, especially in a high HIV-positive population.

Patients and methods: All patients who were treated for spinal TB and underwent an 18F-FDG PET/CT scan were considered for inclusion. PET/CT positive patients underwent a spinal biopsy which was sent for microscopy, Gram staining, Gene Xpert (GXP) polymerase chain reaction (PCR) and histology. Patients in the PET/CT positive group underwent a repeat MRI scan and biopsy at the completion of treatment to investigate the potential presence of resistance or ongoing active spinal TB.

Results: A total of 18 patients were included in the study: five patients were allocated to the PET/CT positive group and 13 to the PET/CT negative group. The PET/CT negative group was significantly older $(p=0.016)$ and had significantly fewer TB-infected vertebrae $(p=0.010)$ than the PET/CT positive group. Two patients, one in each group, were found to have drug-resistant spinal TB. At the 12-month follow-up visit, two patients (40\%) in the PET/CT positive group and three patients (30\%) in the PET/CT negative group were still complaining of back pain. All smear microscopy results of the PET/CT positive patients who underwent a repeat biopsy were negative after the conclusion of treatment; culture results $(n=4 / 4)$ were also negative. GXP PCR results were positive in four and negative in one case. Only one of four samples showed classic TB signs on histology.

Conclusion: This study is the first to report on biopsies done from a PET/CT positive site, after 12 months of anti-tubercular treatment. It is not unlikely that PET/CT is over-sensitive and can show metabolic activity in areas of sterile inflammation, and future studies are necessary to evaluate this.
\end{abstract}

Level of evidence: Level 3

Keywords: PET/CT, spinal tuberculosis, Gene Xpert, histology, tuberculosis

Citation: Davis JH, Burger MC, Pienaar G, Lamberts RP. 18F-FDG PET/CT as a modality for the evaluation of persisting raised infective markers in patients with spinal tuberculosis. SA Orthop J 2020;19(1):23-27. http://dx.doi.org/10.17159/2309-8309/2020/v19n1a3

Editor: Prof. R Dunn, University of Cape Town, South Africa

Received: March 2019

Accepted: October 2019

Published: March 2020

Copyright: @ 2020 Davis JH, et al. This is an open-access article distributed under the terms of the Creative Commons Attribution Licence, which permits unrestricted use, distribution and reproduction in any medium, provided the original author and source are credited.

Funding: No funding was received for this study.

Conflict of interest: The authors declare no conflict of interest with respect to this study. 


\section{Introduction}

The diagnosis of spinal tuberculosis (TB) can be very challenging, with no single test being available that provides $100 \%$ sensitivity in detecting either lung or musculoskeletal TB. ${ }^{1}$ Due to its insidious onset, late presentation and late diagnosis ranging from weeks to years, spinal TB often results in significant levels of morbidity and mortality. ${ }^{2-4}$ Besides having a high index of suspicion of TB spine and performing a thorough clinical assessment, several haematological, microbiological and radiological investigations can be performed to confirm the diagnosis of TB spine.

In addition to these investigations, several blood markers can be used to aid in the diagnosis of TB infection. A popular blood marker which is used for this purpose is the erythrocyte sedimentation rate (ESR), which is an inexpensive and simple blood test that is able to reflect a chronic inflammation state of the human body. ${ }^{5}$ However, the use of ESR as a screening test in asymptomatic persons is limited by its low sensitivity and specificity, ${ }^{5,6}$ and ESR levels can be raised in many other chronic disease processes. A good example of this is in patients with HIV infection, who, due also to a weaker immune system, have a 20 -fold increased risk of contracting TB. ${ }^{7}$

Other cytological, microbiological and DNA-based tests are also used in the diagnosis of TB spine. To confirm the diagnosis of spinal $T B$, it is necessary to either demonstrate acid-fast bacilli (AFB) on microscopy, grow bacilli in culture or have histological evidence of granulomatous inflammation. ${ }^{8}$ Tissue is obtained from diseased vertebrae using a percutaneous needle biopsy or open surgery with the diagnosis of TB spine through histological studies being positive in approximately $60 \%$ of patients. ${ }^{9}$ Additional laboratory tests include Gene Xpert (GXP) polymerase chain reaction (PCR) which is utilised in the molecular testing for TB and which allows direct detection, identification and susceptibility testing, with a reduction in the diagnostic time from weeks to hours. ${ }^{10}$ Because this is a DNA-based test, a false-positive result may be obtained from a repeated biopsy from a site of previous infection. It does, however, confirm that the tissue collected in a repeat biopsy for example, was taken from an area with previously diseased bone. Studies have reported sensitivity of PCR ranging from 61 to $96 \%$ and a specificity of 80 to $98 \% .^{9,11}$ In addition, HIV status can affect this testing method by decreasing its sensitivity. ${ }^{11}$

In terms of radiological assessments, plain film radiographs are the initial investigation for patients suspected of having TB spine. Compared to plain film X-rays, computer tomography (CT) is superior in showing the pathological bony changes ${ }^{12}$ while magnetic resonance imaging (MRI) is regarded as the gold standard imaging modality for spinal infections, with a sensitivity of $96 \%$ and specificity of $94 \% .^{5}$ Abscess collections, together with granulation tissue adjacent to the vertebral body, are highly suggestive of spinal TB, ${ }^{9}$ but there is no pathognomonic finding on MRI that reliably distinguishes TB from other spinal infections or from a possible neoplasm. ${ }^{9}$ In addition to this, walled-off sterile fluid collections can persist in previously diseased bone, following on completion of anti-tuberculous therapy. Apart from minimal oedema and reaction in the adjacent bone, it is very difficult to distinguish from active disease and is not necessarily indicative of on-going TB infection. ${ }^{13}$ Finally, 18-fluorodeoxyglucose positron emission tomography/computer tomography (18F-FDG PET/CT) scan has emerged as a significant molecular imaging technique in clinical oncology and cancer research. ${ }^{14}$ Further, recent literature suggests that the 18F-FDG PET/CT scan can be used as a functional imaging technique to evaluate the extent of spinal TB, and can also be used to assess response to treatment. ${ }^{7,15}$ Zinn et al. ${ }^{7}$ concluded in their work that $18 \mathrm{~F}-\mathrm{FDG}$ PET/CT appears to be a very promising imaging modality in spinal TB, especially for identifying the most appropriate biopsy site, evaluating the disease extent, predicting possible multidrug resistance and monitoring the response to treatment.
Current standard operating procedures prescribe a 12-18 month treatment regimen for spinal TB, which should be monitored through a serial investigation with radiographs and changes in ESR levels and subjective clinical parameters. ${ }^{16}$ In the setting of an HIV pandemic, we are often faced with patients still showing a persisting raised ESR near completion of treatment, posing the question of persisting/resistant spinal TB, HIV co-infection contributing to elevated ESR levels or an alternate diagnosis. Therefore, the aim of the study was to investigate the differences in participant characteristics between positive and negative 18F-FDG PET/CT activity at the spinal TB site following 12 months of the appropriate chemotherapy therapy for spinal TB. A secondary aim of the study was to determine whether ESR levels could be used as a reliable marker of TB activity and/or treatment success of spinal $\mathrm{TB}$, especially in a high HIV-positive population.

\section{Patients and methods}

\section{General characteristics}

All patients who were treated for spinal TB at the Orthopaedic Spinal Unit at Tygerberg Hospital and underwent an 18F-FDG PET/CT scan between January 2012 and December 2014 were considered for inclusion in this retrospective study. Patient records were reviewed and all patients who were a) older than 18 years with spinal TB; b) HIV negative or HIV positive with a CD4 count >200; and had c) confirmed spinal TB by means of tissue biopsy were included in this study. Patients with incomplete data sets or who were lost to follow-up were excluded. Patient records were reviewed, and general and clinical characteristics were recorded for each patient.

\section{Clinical characteristics}

Clinical characteristics from each patient's first presentation to the unit were recorded and all patients underwent an 18F-FDG PET/CT scan. Results were recorded as being PET/CT positive if the scan showed increased metabolic activity at the site of previous spinal TB, considered suggestive of ongoing active spinal TB. Scans that showed no activity were recorded as PET/CT negative. All patients with a PET/CT positive result underwent a repeat MRI scan done to plan the repeat spinal biopsy. Biopsies were sent for microscopy, Gram staining, GXP PCR and histology.

The biopsy method used, and results from smear microscopy, culture, GXP PCR and histology were recorded. Patients in the $\mathrm{PET} / \mathrm{CT}$ positive group underwent a repeat MRI scan and biopsy at the completion of treatment to investigate the potential presence of resistance or ongoing active spinal TB.

\section{Treatment}

All patients were treated with a four-drug anti-tubercular chemotherapy regimen for the complete remainder of the course, without de-escalating after two months. Dose was given as per body weight, and patients were referred to their local clinics to continue their treatment. Data collection from each three-monthly follow-up visit to our spinal clinic included: presence of pain, neurological function score (Frankel grade) and ESR level (using the Westergren method).

\section{Statistical analysis}

The data was analysed using Statistica version 12.0 (StatSoft Inc, Tulsa, OK, USA). The Kolmogorov-Smirnov test was used to test the distribution of the data. Due to the small sample size, data is 
Table I: General characteristics of patients in the PET/CT positive and PET/CT negative groups

\begin{tabular}{|c|c|c|}
\hline Variable & PET/CT positive $(n=5)$ & PET/CT negative $(n=13)$ \\
\hline Age (years) & $29(26-37)^{*}$ & $49(40-54)^{*}$ \\
\hline Sex (male / female) & $2(40 \%) / 3(60 \%)$ & $2(15 \%) / 11(85 \%)$ \\
\hline HIV status (positive / negative) & $4(80 \%) / 1(20 \%)$ & $8(62 \%) / 5(38 \%)$ \\
\hline CD4 count (HIV+ patients) & $491(316-561)$ & $249(155-491)$ \\
\hline Complaints of back pain before the diagnosis (months) & $4(3-6)$ & $4(3-6)$ \\
\hline Number of infected vertebra with TB & $6(4-7)^{* *}$ & $2(2-3)^{* *}$ \\
\hline Location of infection (thoracic / lumbar) & 2 (40\%) / $3(60 \%)$ & $5(38 \%) / 8(62 \%)$ \\
\hline
\end{tabular}

Data is presented as median (interquartile ranges) or frequency with the number of participants in parentheses; ${ }^{*} p=0.016 ;{ }^{* *} p=0.010$

Table II: Biopsy results of PET/CT positive and PET/CT negative patients

\begin{tabular}{|c|c|c|c|c|}
\hline \multirow[t]{2}{*}{ Variable } & \multicolumn{2}{|c|}{ PET/CT positive $(n=5)$} & \multicolumn{2}{|c|}{ PET/CT negative $(n=13)$} \\
\hline & Positive & Negative & Positive & Negative \\
\hline Smear microscopy & $2(40 \%)$ & $3(60 \%)$ & 3 (23\%) & $10(77 \%)$ \\
\hline Culture & $4(80 \%)$ & $1(20 \%)$ & $9(69 \%)$ & $4(31 \%)$ \\
\hline Gene Xpert PCR & $5(100 \%)$ & $0(0 \%)$ & $10(100 \%)^{*}$ & $0(0 \%)^{*}$ \\
\hline & Sensitive & Resistant & Sensitive & Resistant \\
\hline DST 1 & $4(80 \%)$ & $1(20 \%)$ & $9(90 \%)^{*}$ & $1(10 \%)^{*}$ \\
\hline
\end{tabular}

*Only performed in 10 of the 13 patients

PCR: polymerase chain reaction; DST 1: drug susceptibility testing

expressed as medians with interquartile ranges for continuous data while categorical data is expressed as frequencies with the number of participants indicated. Differences between PET/CT positive and PET/CT negative patients were analysed with a Mann-Whitney $U$ test for any statistical significance $(p<0.05$ was considered to be significant).

\section{Results}

\section{General characteristics}

A total of 18 patients were included in the study, with five patients allocated to the PET/CT positive group and 13 to the PET/CT negative group. The PET/CT negative group was significantly older $(p=0.016)$ and had significantly fewer TB-infected vertebrae $(p=0.010)$ than the PET/CT positive group (Table I). Eight patients (44\%) had previous pulmonary tuberculosis (PTB), of which three patients were in the PET/CT positive group and five patients in the PET/CT negative group. Of those with previous PTB, five (63\%) were on anti-tubercular treatment when the spinal TB was diagnosed, with an average treatment duration of three months.

A difference in the neurological function between the two groups at the time of spinal TB diagnosis was observed: all PET/CT positive patients were ambulatory, with four of five $(80 \%)$ having no neurological fallout (Frankel grade E) compared to four out of $12(31 \%)$ of PET/CT negative patients who presented ambulatory (Frankel grade D/E), with the rest (69\%) presenting with neurological fallout that prevented mobilisation.

\section{Pre-treatment outcomes}

All patients underwent an initial biopsy to confirm spinal TB, and biopsy test results from smear microscopy, culture and GXP PCR are described in Table II. Two patients, one in each group, were found to have drug-resistant spinal TB.

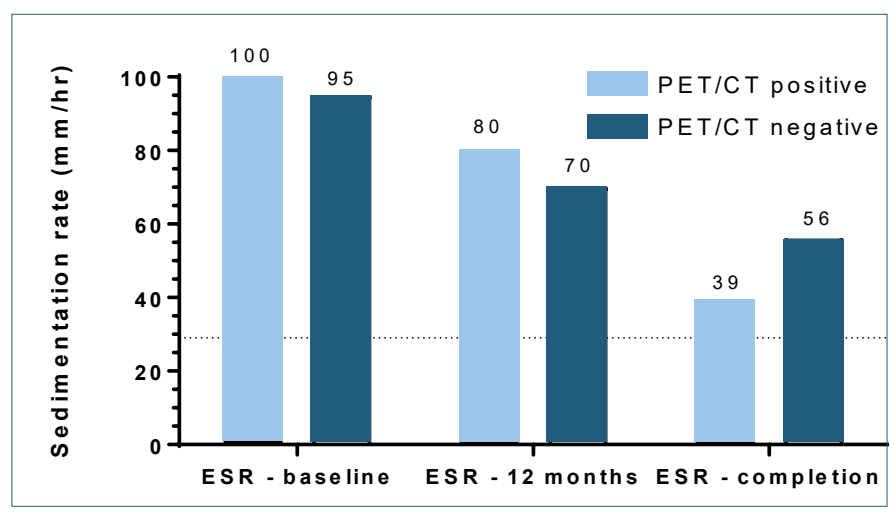

Figure 1. Change in ESR values from admission (ESR - 0), at 12 months (ESR - 12 months) and at completion of treatment (ESR - completion) between PET/CT positive (light bars) and PET/CT negative (dark bars) groups. The shaded area represents the range of normal ESR values. Dotted line: $29 \mathrm{~mm} / \mathrm{hr}$

\section{Treatment outcomes}

At the 12-month follow-up visit, two patients (40\%) in the PET/CT positive group and three patients (30\%) in the PET/CT negative group were still complaining of back pain. ESR values decreased from onset of treatment until completion of treatment but no correlation between the two groups was observed (Figure 1).

All patients in the PET/CT positive group were neurologically intact both at 12 months and at completion of treatment (mean duration of 24 [range 23-28] months). In the PET/CT negative group, $92 \%$ of patients $(n=12 / 13)$ could ambulate at both 12 months and completion of treatment (mean duration of 15 [range 12-18] months). Only one patient was non-ambulatory at completion of treatment (Frankel grade C).

All smear microscopy results of the PET/CT positive patients who underwent a repeat biopsy were negative after the conclusion of treatment and all culture results $(n=4 / 4)$ were also negative. GXP 
Table III: Repeat biopsy results of initial PET/CT positive patients

\begin{tabular}{|l|c|c|}
\hline \multirow{2}{*}{\multicolumn{1}{c|}{ Variable }} & \multicolumn{2}{c|}{ PET/CT (n=5) } \\
\cline { 2 - 3 } & Positive & Negative \\
\hline Smear microscopy & $0(0 \%)$ & $5(100 \%)$ \\
\hline Culture* $^{*}$ & $0(0 \%)$ & $4(80 \%)$ \\
\hline Gene Xpert PCR & $4(80 \%)$ & $1(20 \%)$ \\
\hline & Classic TB signs & Suggestive of TB \\
\hline Histology** & $1(20 \%)$ & $3(60 \%)$ \\
\hline Data is presented as count with frequency indicated in parentheses \\
*one sample not sent for culture; * one sample not sent for histology
\end{tabular}

PCR results were positive in four cases and negative in one. Finally, only one of four samples showed classic TB signs on histology (Table III).

\section{Discussion}

The primary aim of this study was to investigate differences in participant characteristics between patients with a positive and negative 18F-FDG PET/CT scan at the spinal TB site following 12 months of appropriate chemotherapy. Eighteen patients who underwent an 18F-FDG PET/CT scan between January 2012 and December 2014 were included in this study.

The main finding of this study was that participants with a positive PET/CT scan were significantly younger, and had significantly more TB-infected vertebrae than those with a negative result. The latter finding is in agreement with the literature which reports that increased FDG uptake is recorded in active TB in various anatomical locations. ${ }^{17}$ It has further been reported that patients with three or more vertebral lesions affected demonstrated intense FDG uptake, almost three times more that of a single spinal lesion. ${ }^{7}$ Additionally, in the present study, most participants (80\%) in the positive PET/CT scan group were HIV positive, with the majority $(60 \%)$ having had a history of previous PTB. The relationship between HIV and TB is well known and often described as a co-epidemic, and in 2014 the WHO estimated the risk of contracting TB to be 26 to 31 times greater in HIV-positive individuals. ${ }^{18}$

Early diagnosis of spinal TB and appropriate treatment, as per drug sensitivity testing, are the fundamentals in preventing complications. The diagnosis of spinal TB is, however, frequently missed due to insidious onset of the disease and slow development of clinical features. The delay in making the diagnosis spans several months, but can take from several weeks up to years from the onset of symptoms. ${ }^{19,20}$ Spinal TB has been called the great mimicker due to its ability to mimic neoplasms and other atypical infections, especially in HIV-exposed patients. ${ }^{21,22}$ For this reason, microbiological and/or histological confirmation is mandatory for the final diagnosis. The international figures of a positive $M$. tuberculosis culture yield through a percutaneous core needle biopsy is between 50 and $83 \%$, with an average time period of four weeks to obtain the results. ${ }^{23,24}$ Held et al., using the new, rapid molecular diagnostic test (GXP), reported a sensitivity of $95.6 \%$ and specificity of $96.2 \%$, with these results available in 48 hours. ${ }^{23}$ Biopsy results in the present study showed a culture yield in line with international figures while the GXP test was $100 \%$ sensitive in our study, with $10 \%$ of PET/CT negative participants having a positive GXP result. All PET/CT positive patients underwent a repeat spinal biopsy at the site dictated by an MRI scan, to exclude possible new, resistant or on-going infection. None of these showed any TB on microscopy or culture; however, GXP was positive in four of five cases. Considering that GXP is a DNA-based test, it is important to keep in mind that it can lead to false positives, indicating either ongoing active or previous disease. The only way of diagnosing ongoing disease would be through histology showing necrotising granulomatous formation. In the present study, histology of only one repeat biopsy revealed classic necrotising granulomatous inflammation while three cases were suggestive of TB, showing non-necrotising granulomatous inflammation, typically seen in HIVpositive patients.

We further report multi-drug resistant TB (MDR-TB) in both PET/CT groups that gives an overall resistance rate of $13 \%$ for this study compared to other Cape Town-based studies published by Held et al. ${ }^{23}$ and Watt et al. ${ }^{24}$ which reported MDR-TB in $5.8 \%$ and $12 \%$ of patients, respectively. Though overall ESR levels in the present study decreased from diagnosis to 12 months and eventually to completion of treatment, they were still on average above $40 \mathrm{~mm} / \mathrm{hr}$ at the final measurement. A prospective observational study performed in India between 2009 and $2013^{25}$ reported no significant correlation between the ESR levels over time, as measured at presentation and then again at 6,12 and 18 months, and concluded that ESR does not provide any objective evidence of response to treatment. This is in agreement with the findings of the current study, which highlights that this blood marker is unreliable when used as a response monitoring tool, especially in patients with HIV co-infection.

Neurological complications in spinal TB have a prevalence of between 10 and $42 \% .{ }^{26}$ The spinal cord and cauda equina are more tolerable to compression as encountered in TB infection, especially if it happens gradually, and up to $76 \%$ of canal compromise can be tolerated without any neurological compromise. However, paraplegia can develop at lesser canal compromise if mechanical instability or vascular ischaemia is present. ${ }^{27}$ We report half of all the patients being non-ambulatory before the start of treatment. Interestingly these non-ambulatory patients were all in the PET/CT negative group. At the completion of treatment, $92 \%$ of all participants were able to mobilise, without crutches. This finding is echoed in the literature: an average improvement in neurological function by two Frankel grading scores was reported after completion of anti-tubercular treatment. ${ }^{28}$

The duration of treatment was considerably long in both groups, which is concerning considering the risk of drug resistance, patient compliance and drug-related side-effects. The most common reported side-effects are nausea/vomiting, abdominal pain and gastric upset, which disappear after prolonged drug use; however, we saw two cases of optic neuritis due to ethambutol treatment which only partially recovered after changing the drug regimen. Logistical reasons, mainly long waiting periods for appointments for PET/CT scans and follow-up consultations, are likely to blame for the prolonged treatment in the PET/CT negative group.

The main limitation of the study is the retrospective study design. Poor data collection during patient follow-up led to incomplete datasets for several patients, who had to be excluded, subsequently leading to a small sample size. In addition, patients were not all screened for hypo-albuminaemia or anaemia, which could potentially have an effect on the ESR levels.

\section{Conclusion}

This study is the first to report on biopsies done from a PET/CT positive site, after 12 months of anti-tubercular treatment. It might well be that PET/CT is over-sensitive and can show metabolic activity in areas of sterile inflammation, and future studies are necessary to evaluate this.

\section{Ethics statement}

The author/s declare that this submission is in accordance with the principles laid down by the Responsible Research Publication Position Statements as developed at the 2nd World Conference on Research Integrity in Singapore, 2010. Prior to commencement 
of the study ethical approval was obtained from the following ethical review board: Stellenbosch University Health Research Ethics committee, S15/08/174).

Formal consent was not required for this study. All procedures performed in studies involving human participants were in accordance with the ethical standards of the institutional and/or national research committee and with the 1964 Helsinki declaration and its later amendments or comparable ethical standards.

\section{Declaration}

The authors declare authorship of this article and that they have followed sound scientific research practice. This research is original and does not transgress plagiarism policies.

\section{Author contributions}

JHD conceptualised the work and contributed to design, interpretation and drafting of the work. Further contributions include critical revision and expression of intellectual content, as well as final approval of the version submitted to the journal.

MCB contributed to design of the work, analysis and interpretation of the data, drafting as well as revision and final approval.

GP contributed through design of the work and acquisition of data. Interpretation of the data was a further contribution, with final approval of the work prior to submission. RPL contributed to interpretation of the data and statistical analysis as well as approval of the final version.

\section{ORCID}

Davis JH (D) http://orcid.org/0000-0002-1909-7629

Burger MC (D) http://orcid.org/0000-0003-2831-4960

Pienaar G (D) http://orcid.org/0000-0003-3925-1264

Lamberts RP (D) http://orcid.org/0000-0003-1112-2604

\section{References}

1. Watt JP, Davis JH. Percutaneous core needle biopsies: the yield in spinal tuberculosis. South African Med J. 2014;104(1):29-32. doi:10.7196/SAMJ.6868.

2. Ansari S, Amanullah MF, Ahmad K, Rauniyar RK. Pott's spine: diagnostic imaging modalities and technology advancements. $N$ Am J Med Sci. 2013;5(7):404-11. doi:10.4103/1947-2714.115775.

3. Cormican L, Hammal R, Messenger J, Milburn HJ. Current difficulties in the diagnosis and management of spinal tuberculosis. Postgrad Med J. 2006;82:46-51. doi:10.1136/pgmj.2005.032862.

4. Pertuiset E, Beaudreuil J, Liote F, et al. Spinal TB in adults. a study of 103 cases in a developed country, 1980-1994. Medicine (Baltimore). 1999;78(5):309-20.

5. Duarte RM, Vaccaro AR. Spinal infection: State of the art and management algorithm. Eur Spine J. 2013;22(12):2787-99. doi:10.1007/s00586-013-2850-1.

6. Brigden ML. Clinical utility of the erythrocyte sedimentation rate Am Fam Physician. 1999;60(5):1-6.

7. Zinn C, Vorster M, Sathekge MM. Spinal tuberculosis evaluated by means of 18F-FDG PET/CT: Pilot study. Open Nucl Med J. 2014;6(1):6-11. doi:10.2174/1876388X01406010006.

8. Patwardhan SA, Joshi S. Laboratory diagnosis of spinal tuberculosis: past and present. ArgoSpine News J. 2011;23(Sep):120-21. doi:10.1007/s12240-011-0023-9.

9. Garg RK, Somvanshi DS. Spinal tuberculosis: A review. J Spinal Cord Med. 2011;34(5):440-54. doi:10.1179/20457723 11Y.0000000023.

10. Soini H, Musser JM. Molecular diagnosis of mycobacteria. Clin Chem. 2001;47(5):809-814

11. Boehme CC, Nabeta P, Hillemann D, et al. Rapid molecular detection of tuberculosis and rifampin resistance. $N$ Engl $\mathrm{J}$ Med. 2010;363(11):1005-1015.

12. Burrill J, Williams FCJ, Bain FG, Conder FG, Hine FAL, Rakesh R. Tuberculosis: a radiologic review 1. Radiographics. 2007 Sep-Oct;27(5):1255-74.

13. Chaudhary K, Dhawale A, Chaddha R, Laheri V. Spinal tuberculosis - an update. J Clin Orthop. 2017;2(1):31-42. doi:10.13107/jcorth.2456-6993.189.

14. Sobhani I, Tiret E, Lebtahi $\mathrm{R}$, et al. Early detection of recurrence by 18 FDG-PET in the follow-up of patients with colorectal cancer. $\mathrm{Br}$ J Cancer. 2008;98:875-80. doi:10.1038/sj.bjc.6604263.

15. Özmen Ö, Gökçek A, Tatcı E, Biner İ, Akkalyoncu B. Integration of $\mathrm{PET} / \mathrm{CT}$ in current diagnostic and response evaluation methods in patients with tuberculosis. Nucl Med Mol Imaging. 2014;48:75-78. doi:10.1007/s13139-013-0236-2.

16. Jain AK. Tuberculosis of the spine: $A$ fresh look at an old disease. J Bone Jt Surg. 2010;92(7):905-13. doi:10.1302/0301-620X.92B7.24668.

17. Harkirat S, Anana SS, Indrajit LK, Dash AK. Pictorial essay: PET/CT in tuberculosis. Indian J Radiol Imaging2. 2008;18(2):141-47.

18. WHO. Global Tuberculosis Report 2015. doi:WHO/HTM/ $\mathrm{TB} / 2014.08$.

19. Rivas-Garcia A, Sarria-Estrada S, Torrents-Odin C, Casas-Gomila L, Franquet E. Imaging findings of Pott's disease. Eur Spine J. 2013;22:567-78. doi:10.1007/s00586-012-2333-9.

20. Arciniegas W, Orjuela DL. Extrapulmonary tuberculosis: A review of 102 cases in Pereira, Colombia. Biomedica. 2006;19:71-80.

21. D'souza MM, Mondal A, Sharma R, Jaimini A, Khanna U. Tuberculosis the great mimicker: 18F-fludeoxyglucose positron emission tomography/computed tomography in a case of atypical spinal tuberculosis. Indian J Nucl Med. 2014;29(2):99-101. doi:10.4103/0972-3919.130294.

22. Dunn R. The medical management of spinal tuberculosis. $S A$ Orthop J. 2010;9(1):37-41.

23. Held M, Laubscher M, Zar HJ, Dunn RN. Gene Xpert polymerase chain reaction for spinal tuberculosis: an accurate and rapid diagnostic test. Bone Joint J. 2014;96-B(10):1366-1369. doi:10.1302/0301-620X.96B10.34048

24. Watt JP, Davis JH. Percutaneous core needle biopsies: The yield in spinal tuberculosis. South African Med J. 2014;104(1):29-32. doi:10.7196/SAMJ.6868.

25. Dureja S, Sen IB, Acharya S. Potential role of F18 FDG PET-CT as an imaging biomarker for the noninvasive evaluation in uncomplicated skeletal tuberculosis: a prospective clinical observational study. Eur Spine J. 2014;23(11):2449-54. doi:10.1007/s00586-014-3483-8.

26. Adendorff JJ, Boeke EJ, Lazarus C. Pott's paraplegia. South African Med J. 1987;71(7):427-28.

27. Jain AK, Aggarwal A, Mehrotra G. Correlation of canal encroachment with neurological deficit in tuberculosis of the spine. Int Orthop. 1999;23(2):85-86. doi:10.1007/s002640050313.

28. Dunn R, Zondagh I, Candy S. Spinal tuberculosis: magnetic resonance imaging and neurological impairment. Spine (Phila Pa 1976). 2011;36(6):469-73 